\title{
ALTERATIONS TO THE CENSUS OF NEW SOUTH WALES PLANTS
}

\author{
S. W. L. JACOBS \& L. LAPINPURO
}

(Accepted for publication 11.4.1984)

\begin{abstract}
Jacobs, S. W. L. \& Lapinpuro, L. (National Herbarium of New South Wales, Royal Botanic Gardens, Sydney, Australia 2000) 1986. Alterations to the Census of New South Wales plants. Telopea 2(6): 705-714 - Corrections and additions are made to Jacobs \& Pickard, Plants of New South Wales, (1981) to update the information presented in that publication.
\end{abstract}

\section{INTRODUCTION}

Future corrections and additions were forecast in the original publication of a census of New South Wales plants (Jacobs \& Pickard 1981). This present paper is the first attempt to update the information.

The alterations are mainly concerned with information for New South Wales, the principal concern of the Census, and corrections for extra-New South Wales distributions are only made where alterations are being made to the New South Wales information.

Alterations have been incorporated where we have been notified, through the taxonomic botanist responsible for the curation of the family, of any taxonomic or geographic inaccuracies. While geographic alterations tend to flow through fairly rapidly, there is usually some delay before a taxonomic treatment is evaluated by the taxonomist curating the group and its acceptance communicated. The length of these delays is quite variable, even for one botanist. Thus the omission of any taxonomic treatment in these alterations is in no way a comment by us to its acceptance and adoption by our institution.

The same abbreviations are used as in the original publications. As before, the arrangement is alphabetical with monocotyledons preceding dicotyledons. Deletion of the entry for a family or genus implies deletion of all names included therein.

In accordance with a recent change in the International Code of Botanical Nomenclature, epithets ending in '-eranus', '-erana' and '-eranum' should be altered to '-erianus', '-eriana' and '-erianum' respectively, e.g. hookerianus, forsteriana, billardierianum.

\section{ACKNOWLEDGMENTS}

We wish to thank our colleagues at the National Herbarium who have kept us fed with alterations; in particular we would like to thank Teresa James, Marie Taylor, Karen Wilson and Joy Everett. We would also like to thank Bill 
Mulham for drawing our attention to many extensions of range in the south-western areas of the State, and Dr J. H. Willis for his helpful list of corrections.

\section{REFERENCE}

Jacobs, S. W. L. \& Pickard, J. (1981). 'Plants of New South Wales' (Govt Printer: Sydney).

\section{ALTERATIONS AND ADDITIONS}

\section{ALLIACEAE}

Allium

add: *A. neapolitanum Cyrillo; ${ }^{\dagger} \mathrm{CWS},{ }^{\dagger} \mathrm{SWS}$; .S; Medit

\section{AMARYLLIDACEAE}

delete Eurycles Salisb. and replace by:

PROIPHYS Lindl.

P. cunninghamii (Lindl.) Mabberley

\section{CENTROLEPIDACEAE \\ Centrolepis \\ C. polygyna add: SWP \\ C. strigosa add: SWP}

\section{CYPERACEAE}

Baumea

delete: $B$. sp. C and replace by:

B. johnsonii $\mathrm{K}$. L. Wilson

add: BOLBOSCHOENUS (Aschers.) Palla

B. caldwellii (V. J. Cook) Soják; NC, CC, SC, ST, CWS, SWS, NWP; VTSW; NZ

[Scirpus caldwellii V. J. Cook]

B. fluviatilis (Torrey) Soják; NC, CC, SC; Q; NZ, Asia, N Amer

[Scirpus maritimus L. var. Juviatilis Torrey, $S$. fluviatilis (Torrey) A. Gray]

B. medianus (V. J. Cook) Soják; CC, NT, ST, CWS, NWP, SFWP; VTS; NZ, NG

[Scirpus medianus V. J. Cook]

Carex

delete: $C$. curta Good. and replace by:

C. canescens $L$.

[C. curta Good.]

Caustis

C. flexuosa add: NC

Cyperus

C. compressus add: ${ }^{\dagger} \mathrm{NC}$

add: ${ }^{\dagger}$ C. dubius Rottb.; ${ }^{\dagger} \mathrm{NC}$; trop, S Afr

[Mariscus dubius (Rottb.) Kükenthal ex Fischer]

C. eragrostis add: $₫ \mathrm{NWS}$

C. exaltatus add: SFWP

C. gunnii add: NT

C. polystachyos add: ?*

C. sanguinolentus add: CWS, SWS

C. tenellus add: ?*; SWP

C. unioloides add: NC, ?ST

Fimbristylis

$F$. nutans add: CC

Gahnia

G. radula add: NC add: ISOLEPIS R.Br.

I. aucklandica Hook. f.; NT, ST; QVT; NG, NZ

[Scirpus aucklandicus (Hook. f.) Boeck.]

I. australiensis (Maiden \& Betche) K. L. Wilson; NT, CT, CWS, NWP, NFWP; QVYS; ?NZ

[Scirpus cernuus var. australiensis Maiden \& Betche, $S$. australiensis (Maiden \& Betche) S. T. Blake, S. multicaulis F. Muell. non Sm. nec Gmel. nec Schltdl.]

I. cernua (Vahl) Roem. \& Schult.; NC, CC, SC, CT, ST, CWS; QVTSW; cosmop [Scirpus cernuus Vahl, S. psammophilus S. T. Blakel

I. congrua Nees; CWS, NWP, SWP, NFWP: VYSW

[Scirpus congrua (Nees) S. T. Blake,

S. kochii Maiden \& Betche]

I. crassiuscula Hook. f.; NT, ST; QVT; NG, NZ, Japan [Scirpus crassiusculus (Hook. f.) Benth. Eleogiton crassiusculus (Hook. f.) Soják

I. fluitans (L.) R. Br.; CC, SC, NT, ST; VTSW; Eur, Afr, Asia, NZ

[Scirpus fluitans L., S. lenticularis Poir.]

I. habra (E. Edgar) Soják; NT, CT, ST, SWS; VT; NZ, ?NG

[Scirpus habrus E. Edgar]

I. hookeriana Boeck.; CC, NT, CT, ST, NWS SWS, NWP, SWP; QVTSW; NZ

[Scirpus hookerianus (Boeck.) S. T. Blake, $S$. colocarpus S. T. Blake, S. setaceus auct. austr. non L.]

*I. hystrix (Thunb.) Nees; ${ }^{\dagger}$ SWP; V; S Afr [Scirpus hystrix Thunb.]

I. inundata R. Br.; NC, CC, SC, NT, CT, ST, SWS; QVTSW; S Amer, Malesia [Scirpus inundatus (R. Br.) Poir. S inundatus var. floribundus Benth., $S$. inundatus f. urvillei (Boeck.) Kükenthal

I. marginata (Thunb.) A. Dietr.; CC, CT, ST, CWS, SWP, SFWP; VTSW; NZ, S Afr [Scirpus marginatus Thunb., S. antarcticus auct. non L., S. cartilagineus (R. Br.) Poir.]

I. montivaga (S. T. Blake) K. L. Wilson; ST; V [Scirpus montivagus S. T. Blake]

I. nodosa (Rottb.) R. Br.; LHI, NC, CC, SC; QVTSW; temp S hemi

[Scirpus nodosus Rottb., Holoschoenus nodosus (Rottb.) A. Dietr.]

I. platycarpa (S. T. Blake) Soják; CC, ST, SWS; VTSW; NZ [Scirpus platycarpus S. T. Blake] 
1. producta (C. B. Clarke) K. L. Wilson; SC; VTS

[Scirpus productus C. B. Clarke]

*I. prolifera (Rottb.) R. Br.; ${ }^{\dagger} \mathrm{CC},{ }^{\dagger} \mathrm{SC},{ }^{\dagger} \mathrm{CT}$; ?VW; S Afr, NZ

[Scirpus prolifer Rottb.]

*I. sepulcralis Steud.; ${ }^{\dagger} \mathrm{NC},{ }^{\dagger} \mathrm{CC}$; $\mathrm{S}$ Afr, $\mathrm{NZ}$ [Scirpus chlorostachyus Levyns, $S$. sp. sensu Jacobs \& Pickard]

I. stellata (C. B. Clarke) K. L. Wilson; ? ${ }^{\dagger} \mathrm{CC}$; VTSW

[Scirpus stellatus C. B. Clarke]

I. subtilissima Boeck.; NC, SC, NT, CT; QVT;

Malesia, NZ

[Scirpus subtilissimus (Boeck.) S. T. Blake, S. merrillii (Palla) Kükenthal ex Merr.]

I. victoriensis (N. A. Wakef.) K. L. Wilson; CWS, SWS, SWP; V ?S

[Scirpus victoriensis N. A. Wakef.]

Lipocarpha

L. microcephala add: NC

[Rikliella australiensis J. Raynal, Scirpus squarrosus L. var. dietrichiae (Boeck.)

Benth.]

delete: Rikliella

add: SCHOENOPLECTUS Palla

S. dissachanthus (S. T. Blake) J. Raynal; NWP, SWP; QVYSW

[Scirpus dissachanthus S. T. Blake]

*S. erectus (Poir.) Palla ex J. Raynal; ${ }^{\dagger} \mathrm{CC}$, †WP; QV; Afr

[Scirpus raynalii Schuyler, Scirpus lateriflorus Gmel. misappl., Scirpus supinus L. misappl.]

S. laevis (S. T. Blake) J. Raynal; NWP; QYW [Scirpus laevis S. T. Blake]

*S. lineolatus (Franch. \& Sav.) T. Koyama; †CC; V; Japan

[Scirpus lineolatus Franch. \& Sav, Scirpus forsythii Kükenthal]

?*S. litoralis (Schrad.) Palla; NC, CC; QYSW; Eur, Asia

[Scirpus litoralis Schrad.]

S. mucronatus (L.) Palla ex Kerner; NC, CC, NT, NWS; QYW; Eur, Asia

[Scirpus mucronatus L.]

S. pungens (Vahl) Palla; ST, CWS, SWS; VTS; Eur, Amer, NZ

[Scirpus pungens Vahl, Scirpus americanus auct. non Pers.]

S. validus (Vahl) A. Löve \& D. Löve; NC, CC, SC, NT, ST, NWS, SWS, NWP, SFWP;

QVTSW; Amer, NZ, N Caled

[Scirpus validus Vahl, Scirpus lacustris auct. austr. non L.]

Scirpus

delete: all species except $S$. polystachyus F. Muell.

Scleria

S. mackaviensis add: CT

add: TRACHYSTYLIS S. T. Blake

T. stradbrokensis (Domin) Kükenthal; NC; $\mathrm{Q}$

\section{HYPOXIDACEAE}

Hypoxis

delete: $H$. pusilla Hook. f. and replace by:

H. hookeri Geerink

[H. pusilla Hook. f.]

\section{IRIDACEAE}

add: *ANOMATHECA Ker Gawler

*A. laxa (Thunb.) Goldblatt; †CC; S Afr

[Lapeirousia laxa (Thunb.) N.E.Br.]

correct to: Diplarriena

Gladiolus

add: ${ }^{*} \mathbf{G}$. angustus $\mathrm{L} .{ }^{\dagger} \mathrm{CC} ; \mathrm{S} \mathrm{Afr}$

${ }^{*} \mathrm{G}$. carneus Delaroche; ${ }^{\dagger} \mathrm{CC} ; \mathrm{S}$ Afr

delete: $G$. x colvillei

Homeria

add: *H. collina (Thunb.) Salisb.; ${ }^{\dagger} \mathrm{NC},{ }^{\dagger} \mathrm{CC}$,

†SC, †SWS; W; S Afr

$H$. flaccida delete: $\mathrm{NC}$, SWS, SWP

$H$. miniata delete: $C C$; add: SWP

add: ${ }^{\star}$ H. ochroleuca Salisb. ${ }^{\dagger} \mathrm{CC},{ }^{\dagger} \mathrm{SWS},{ }^{\dagger} \mathrm{SWP}$

$\mathrm{S}$; S Afr

Ixia

change $I$. columellaris to:

*I. polystachya L.; $\dagger^{\dagger} C$; S Afr

[I. columellaris Ker Gawler misappl.]

delete: Lapeirousia

Sisyrinchium

S. micranthum add: "NWS

Watsonia

delete: $W$. angusta and replace by:

*W. bulbillifera J. Mathews \& L. Bolus

[W. angusta Ker Gawler misappl.]

JUNCACEAE

Juncus

$J$, acutus add: †CWS

JUNCAGINACEAE

Triglochin

T. calcitrapa add: SFWP

LILIACEAE

delete: Anguillaria

Thysanotus

T. baueri add: SFWP

add: WURMBEA Thunb.

W. biglandulosa (R. Br.) Macfarlane; NC, SC, NT, ST, NWS, CWS, SWS, NWP; V

[Anguillaria biglandulosa $\mathrm{R}$. Br.]

W. dioica (R. Br.) F. Muell.

[Anguillaria dioica $\mathrm{R}$. $\mathrm{Br}$.]

ssp. dioica; CC, NT, CT, ST, NWS, CWS,

SWS, NWP, SWP, NFWP; QVTSW

W. latifolia Macfarlane; ST, CWS, SWS; VS

W. uniflora (R. Br.) Macfarlane; SC

[Anguillaria uniflora $\mathrm{R}$. Br.]

ORCHIDACEAE

Dipodium

D. hamiltonianum add: SWS; V

POACEAE

Amphibromus

replace all species by:

A. fluitans Kirk; SWS, SWP; V; NZ

[A. gracilis P. F. Morris]

A. macrorhinus S. Jacobs \& Lapinpuro; SWS, SWP; VTS

A. nervosa (J. D. Hooker) Druce; CC, NT, CT, ST, NWS, CWS, SWS, NWP, SWP, SFWP; VTSW

[A. neesii auct. non Steud.] 
A. pithogastrus S. Jacobs \& Lapinpuro; NT, CT, ST, SWS; V

[A. sp. A sensu Jacobs \& Pickard]

A. sinuatus S. Jacobs \& Lapinpuro; NT; V

Andropogon

$A$. virginicus add: ${ }^{\dagger} \mathrm{CWS}$

Bromus

delete: ${ }^{*} B$. unioloides Kunth and replace by:

*B. catharticus Vahl

[B. unioloides Kunth, B.willdenowii Kunth]

Chionochloa

add: C. pallida (R. Br.) S. Jacobs; NC, CC, SC,

NT, CT, ST, NWS, CWS, SWS, SWP; V

[Danthonia pallida R. Br., Notodanthonia pallida (R. Br.) Veldkamp]

Danthonia

delete: $D$, pallida $\mathrm{R}$. Br.

Eleusine

$E$. indic $a$ add: ${ }^{\dagger} \mathrm{NWS},{ }^{\dagger} \mathrm{SWP}$

add: *HAINARDIA Greuter

${ }^{*}$ H. cylindrica (Willd.) Greuter; ${ }^{\dagger} \mathrm{LHI},{ }^{\dagger} \mathrm{NC}$, ${ }^{\dagger} \mathrm{CC},{ }^{\dagger} \mathrm{ST},{ }^{\dagger} \mathrm{NWS},{ }^{\dagger} \mathrm{CWS},{ }^{\dagger} \mathrm{SWS},{ }^{\dagger} \mathrm{NWP}$, tSWP; VT; Eur, Amer

[Monerma cylindrica (Willd.) Cosson \&

Durieu]

Homopholis

$H$. belsonii delete: NWP; add: NWS

Hordeum

$H$. leporinum add: ${ }^{\dagger} \mathrm{NT},{ }^{\dagger} \mathrm{CT},{ }^{\dagger} \mathrm{CWS}$

Koeleria

replace: $K$. cristata by:

K. macrantha (Ledeb.) Schultes

[K. cristata (L.) Pers.]

Lophochloa

L. cristata correct to: Hylander

Microlaena

$M$. stipoides var. stipoides add: LHI

delete: Monerma

Panicum

$P$. coloratum var. makarikariense add: ${ }^{\dagger} \mathrm{NC}$

$P$. effusum add: SFWP

Paspalum

$P$. distichum change synonymy to:

[P. paspalodes (Michx.) Scribn.];

change distribution to: LHI, NC, CC, NT

CT, ST, NWS, CWS, NWP, SWP, NFWP QVYSW; trop, subtrop

P. notatum add: ${ }^{\dagger} \mathrm{NWS}$

delete: $P$. paspalodes

add: P. vaginatum Sw.; LHI, NC, CC, SC,

CWS; QYW; trop, subtrop

[P. littorale $\mathrm{R} . \mathrm{Br}$.]

correct to: Pentaschists

$P$. airoides add: ${ }^{\dagger} S W P$

Phalaris

$P$. paradoxa add: ${ }^{\dagger}$ NFWP

Spinifex

replace: $S$. hirsutus by:

S. sericeus R.Br.; LHI, NC, CC, SC; QVTS;

NZ, N Caled

[S.hirsutus auct. non Labill.]

Stipa

This genus is still under revision.
Triraphis

T. mollis add: CWS

POTAMOGETONACEAE

Potamogeton

$P$. ochreatus add: NWS

add: RUPPIA L.

R. maritima L.; NC, SC, SWP; QVYSW; Eur

R. megacarpa $\mathrm{R}$. Mason; NC, CC, SC, ST, SWP; VWS; NZ

$[R$. spiralis auct. non L. ex Dumort.,

$R$. cirrhosa auct. non (Petagna) Grande

R. polycarpa R. Mason; CC, SC; VTSW; NZ

$[R$. spiralis auct. non L. ex Dumort,

$R$. cirrhosa auct. non (Petagna) Grande]

delete: RUPPIACEAE

XANTHORRHOEACEAE

Xanthorrhoea

$X$. australis $\mathrm{ssp}$. australis (western form) add: SWP

$X$. johnsonii

delete: ssp. fulva

delete: ssp. johnsonit

$X$. minor add: ST; VTS; delete: Q

AIZOACEAE

delete: Aizoon

Glinus

G. oppositifolia add: SFWP

add: GUNNIOPSIS Pax

G. quadrifida (F. Muell.) Pax; NFWP; QYSW [Aizoon quadrifidum (F. Muell.) F. Muell.]

G. papillata Chinnock; NFWP; QYS

[Aizoon zygophylloides F. Muell. misappl.]

G. septifraga (F. Muell.) Chinnock; NFWP, SFWP; YSW

[Neogunnia septifraga (F. Muell.) Pax \& K. Hoffm.]

Mollugo

$M$. verticillata add: NWS

delete: Neogunnia

AMARANTHACEAE

Alternanthera

A. angustifolia add: SWP

A. nana add: CWS

Amaranthus

add: *A. cruentus L.; ${ }^{\dagger} \mathrm{CC},{ }^{\dagger} \mathrm{SWS}$; Eur, Amer $A$. retroflexus add: ${ }^{\dagger} \mathrm{NWS}$

Ptilotus

add: P. extenuatus Benl; SWP

APIACEAE

Apium

add: *A. graveolens L.; ${ }^{\dagger} \mathrm{CC}$, ${ }^{\dagger}$ SWP; Y; Eur, Asia, Afr, Amer

Hydrocotyle

H. laxiflora add: SWP

APOCYNACEAE

Alstonia

A. constricta

'pubescent' form add: CWS

Alyxia

A. ruscifolia add: $\mathrm{CC}$ 


\section{ASCLEPIADACEAE}

Tylophora

T. grandiflora add: CWS

\section{ASTERACEAE \\ Actites \\ A. megalocarpa add: LHI \\ Ambrosia \\ A. tenuifolia add: NFWP \\ Angianthus \\ delete: $A$. pusillus}

correct Artemsia to Artemlsia

$A$. arborescens add: † $\$$ WP

Baccharis

$B$. halimifolia add: * $\mathrm{CC}$

Bidens

B. pilosa add: NT

$B$. subalternans add: SWP

Brachycome

B. campylocarpa add: NFWP

GEN. NOV. change to: CAMPTACRA N. Burb.

$C$. sp. change to: $C$. brachycomoides ( $F$. Muell.)

$$
\text { N. Burb.; add: Y }
$$

add: C. barbata N. Burb.; NWS; Q

\section{Carthamus}

$C$. tinctorius add: $\dagger C T$

add: CHRYSOCORYNE Endl.

C. pusilla (Benth.) Endl.; NWS, NWP, SWP, NFWP, SFWP; QVYSW

[Angianthus pusillus (Benth.) Benth.]

Cratystylis

change to: $C$. conocephala; SFWP

Galinsoga

G. parviflora add: †SWP

Gnaphalium

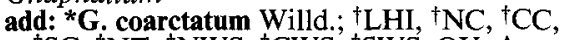

${ }^{\dagger} \mathrm{SC},{ }^{\dagger} \mathrm{NT},{ }^{\dagger} \mathrm{NWS},{ }^{\dagger} \mathrm{CWS},{ }^{\dagger} \mathrm{SWS} ; \mathrm{QV} ;$ Amer

[G. spicatum Lamk.]

delete: G. candidissimum

$G$. indutum add: ?SWP

delete: $G$. luteo-album

delete: G. spicatum

Helianthus

H. annuus add: ${ }^{\dagger} \mathrm{ST}$

Helichrysum

add: H. whitei N. Burb.; NC; Q

Helipterum

H. strictum add: NWS

Hypochoeris

H. glabra add: ${ }^{\dagger} \mathrm{NFWP}$

Olearia

O. calcarea add: SFWP

$O$. rudis var. rudis add: SFWP

$O$. subspicata add: NFWP

Onopordum

O. acaulon add: ${ }^{\dagger}$ NFWP

add: PARTHENIUM L.

P. hysterophorus L.; NWP; Q; Amer, Afr, NZ, N Caled
Picris

$P$. hieracioides

change to: ssp. hieracioides

add: PSEUDOGNAPHALIUM Kirpichnikov

P. luteoalbum (L.) Hilliard \& Burtt; LHI, NC, CC, SC, NT, CT, ST, NWS, CWS, SWS,

NWP, SWP, NFWP, SFWP; QVTYSW

[Gnaphalium luteo-album L.]

Senecio

S. cunninghamii add: ST

add: $S$. georgianus $D_{C}$; ST

delete: $S$. sp. G

delete: $S$. sp. K

Previous record not substantiated

delete: $S$. georgianus

Soliva

S. stolonifera add: ${ }^{\dagger} \mathrm{SWP}$

Sonchus

S. asper ssp. glaucescens add: ${ }^{\dagger} \mathrm{NFWP}$

Tolpis

T. umbellata add: ${ }^{\dagger} \mathrm{NC}$

add: *UROSPERMUM Scop.

*U. picroides (L.) Scop. ex F. W. Schmidt; SWP; VS; Medit

add: *VELLEREOPHYTON Hilliard \& Burtt

*V. dealbatum (Thunb.) Hilliard \& Burtt; ${ }^{\dagger} \mathrm{CC}$; VTS; S Afr

[Gnaphalium candidissimum Lamk.]

Vittadinia

add: V. arida N. Burb.; NFWP; QYSW

V. sp. A change to:

V. cervicularis N. Burb.

var. A change to: var. cervicularis

var. B change to: var. subcervicularis $\mathrm{N}$. Burb.

delete: f. $a, f . b$

$V . \mathrm{sp}$. B change to:

V. condyloides N. Burb.

$V$. cuneata

var. A change to: var. cuneata

$f$. a change to: $f$. minor

f. $b$ change to: $f$. cuneata

var. B change to: var. hirsuta N. Burb.

var. $C$ change to: var. morrisii N. Burb.

$V$. sp. C change to:

V. dissecta N. Burb. var. A change to: var. dissecta

var. $B$ and var. $C$ change to: var. hirta $N$. Burb.

$V$. sp. D change to:

V. eremaea N. Burb.

$V$. sp. E change to:

V. gracilis N. Burb.

$V$. sp. F change to:

V. sulcata N. Burb.

$V$. hispidula

var. hispidula change to: var. setosa N. Burb.

var. A change to: var. hispidula

$V$. sp. G change to:

V. muelleri N. Burb.

$V$. sp. H change to:

V. pustulata N. Burb.

BORAGINACEAE

add: *NEATOSTEMA I. M. Johnston 

*N. apulum (L.) I. M. Johnston; †SFWP; S;
Medit

\section{BRASSICACEAE}

Lepidium

L. campestre add: ${ }^{\dagger} \mathrm{SC}$

CABOMBACEAE

add: *CABOMBA Aubl.

${ }^{*} \mathrm{C}$. caroliniana Gray; ${ }^{\dagger} \mathrm{NC}$, $\nmid \mathrm{CC}$; Amer

CACTACEAE

add: *ERIOCEREUS (A. Berger) Riccob.

*E. martinii (Labouret) Riccob.; ${ }^{\dagger}$ NWP; Amer

*E. tortuosus (J. Forbes ex Otto \& A. Dietr.)

Riccob.; †CWS; Amer

Opuntia

$O$. aurantiaca add: ${ }^{\dagger} \mathrm{CC}$

CAESALPINIACEAE change to: subfamily

Caesalpinioideae (of FABACEAE)

add: *DELONIX Raf.

*D. regia (Bojer) Rafin.; ${ }^{\dagger} \mathrm{NWP}$; Asia, Pac I

CALLITRICHACEAE

add: C. cyclocarpa Hegelm.; SWP; V

C. stagnalis add: NFWP

\section{CARYOPHYLLACEAE \\ Silene \\ delete: $S$. alba \\ add: *S. pratensis (Rafn) Godron \& Gren.; \\ ${ }^{\dagger} \mathrm{NC},{ }^{\dagger} \mathrm{CC},{ }^{\dagger} \mathrm{SC},{ }^{\dagger} \mathrm{NT},{ }^{\dagger} \mathrm{CT},{ }^{\dagger} \mathrm{ST}$; VTS; \\ Eur, W Asia \\ [S. alba (Miller) E. H. L. Krause, Lychnis alba Miller]}

Spergula

$S$. arvensis add: ${ }^{\dagger}$ SWP

add: *S. pentandra L.; ${ }^{\star S W P}$; V; Eur

CASUARINACEAE

add: ALLOCASUARINA L. A. S. Johnson

A. distyla (Vent.) L. A. S. Johnson; NC, CC, SC, CT, ST

[C. distyla Vent.]

A. inophloia (F. Muell. \& F. M. Bail.)

L. A. S. Johnson; NWS; Q

[C. inophloia F. Muell. \& F. M. Bail.]

A. littoralis (Salisb.) L. A. S. Johnson; NC, CC, SC, NT, CT, ST, NWS, CWS, NWP; QVT [C. littoralis Salisb.]

A. luehmannii (R. T. Baker) L. A. S. Johnson; NC, NT, ST, NWS, CWS, SWS, NWP, SWP, SFWP; QVS

[C. huehmannii $\mathrm{R}$. T. Baker]

A. nana (Sieber ex Spreng.) L. A. S. Johnson; CC, SC, CT, ST; V

[C. nana Sieber ex Spreng.]

A. paludosa (Sieber ex Spreng.) L. A. S.

Johnson; CC, SC, CT, ST; VTS

[C. paludosa Sieber ex Spreng.]

A. rigida (Miq.) L. A. S. Johnson; NC, NT NWS; $\mathrm{O}$ [C. rigida $\mathrm{Miq}$.

A. torulosa (Ait.) L. A. S. Johnson; NC, CC, SC, NT, CT, NWS, CWS; Q [C. torulosa Ait.]

A. verticillata (Lamk.) L. A. S. Johnson; CC, SC, CT, ST, CWS, SWS, SWP; VTS [C. verticillata Lamk., C. stricta Ait.]
Casuarina

C. cunninghamiana ssp. cunninghamiana

delete: $\mathrm{NG}$

delete: $C$. distyla

delete: C. inophloia

delete: $C$. littoralis

delete: C. luehmannii

delete: $C$. nana

delete: C. paludosa

delete: $C$. rigida

delete: $C$. stricta

delete: $C$. torulosa

\section{CHENOPODIACEAE}

Atriplex

add: A. nessorhina S. Jacobs; NFWP; Q?YS

[A. sp. C sensu Jacobs \& Pickard]

A. pseudocampanulata add: SFWP

add: A. sturtii S. Jacobs; NFWP; QYS

[A. leptocarpa f. minor R. H. Anderson, $A$.

sp. A sensu Jacobs \& Pickard]

Chenopodium

C. ambrosioides add: ${ }^{\dagger} \mathrm{CWS}$

C. carinatum add: $\mathrm{CT}$

Enchylaena

E. tomentosa form a add: SWP

Halosarcia

add: H. halocnemoides (Nees) P. G. Wilson ssp. longispicata P. G. Wilson; ? NFWP; QYS

H. pergranulata ssp. pergranulata add: SWP add: ssp. divaricata P. G. Wilson; NWP,

NFWP; QVS

add: H. pluriflora P. G. Wilson; NWP; S

Maireana

add: M. rohrlachii (P. G. Wilson) P. G. Wilson; SWP; S

Pachycornia

$P$. triandra add: NWP

Salsola

S. kali var. kall add: NC, ST; Eur

Sclerolaena

S. muricata

add: var. muricata; ${ }^{\dagger} \mathrm{CC}, \mathrm{NWS}, \mathrm{CWS}, \mathrm{NWP}$, SWP, NFWP, SFWP; QVYS

Suaeda

$S$. sp. A change to: ${ }^{*}$. baccifera Pallas add: +SFWP

\section{CONVOLVULACEAE}

Cuscuta

C. campestris add: ${ }^{\dagger} \mathrm{NFWP}$

add: *C. racemosa Mart.; ${ }^{\dagger} S T$; V; Amer

\section{CUNONIACEAE}

delete: Ackama

add: ACROPHYLLUM Benth.

A. australe (A. Cunn.) Hoogl.; CT

$[A$. venosum Benth., Calycomis australis (A. Cunn.) Hoogl.]

add: CALDCLUVIA D. Don

C. paniculosa (F. Muell.) Hoogl.; NC, CC; Q [Weinmannia paniculosa $\mathrm{F}$. Muell., $W$. paniculata F. Muell., Ackama muelleri

Benth., A. paniculata var. hirsuta Maiden \& Betche, $A$. hirsuta (Maiden \& Betche) Cheel] delete: Calycomis 


\section{DILLENIACEAE}

Hibbertia

add: H. procumbens (Labill.) DC.; CC

[H. angustifolia Salisb.]

$H$. serice $a$ add: NWP; T

\section{ELATINACEAE}

Bergia

add: B. perennis (F. Muell.) F. Muell. ex

Benth. form A; NFWP; Y

EPACRIDACEAE

Leucopogon

L. microphyllus var, pilibundus add: CT

EUPHORBIACEAE

Euphorbia

E. dentata add: ${ }^{\dagger} \mathrm{CWS}$

Phyllanthus

$P$. maderaspatanus add: NWS

$P$. tenellus add: ${ }^{\dagger} \mathrm{CT}$

\section{EUPOMATIACEAE}

Eupomatia

E. laurina delete: NWS

FABACEAE

Fabaceae, Caesalpiniaceae and Mimosaceae as treated here should be reduced to subfamilies Faboideae, Caesalpinioideae and Mimosoideae of the family FABACEAE. add: ABRUS Adans.

A. precatorius L.; NC; QY; Asia, Pac I

Bossiaea

add: B. oligosperma A. Lee; CC

Crotalaria

add: ${ }^{*}$ C. grahamiana; add: ${ }^{\dagger} \mathrm{NC}$; India

Dillwynia

add: D. cinerascens R. Br.; SWP; VTSW

Jacksonia

add: J. turnerana Domin; NWP; Q

add: LABLAB Adans.

L. purpureus (L.) Sweet; NC; India [Dolichos lablab L.]

Lupinus

L. cosentinil add: ${ }^{\dagger} \mathrm{NC}$

Macroptilium

add: *M. atropurpureum (DC.) Urban; ${ }^{\dagger} \mathrm{NC}$, ${ }^{\dagger} \mathrm{CC}$; Q; C Amer, Afr

[Phaseolus atropurpureus DC.]

add: *MACROTYLOMA (R. Wight \& Arnott) Verdcourt

*M. axillare (E. Mey.) Verdcourt; ${ }^{\dagger} \mathrm{NC}$; Afr [Dolichos axillaris E. Mey]

Psoralea

add: P. pallida N. Burb.; NWP, SWP, NFWP, SFWP; QVYS

$[P$. sp. (aff. patens) sensu Jacobs \& Pickard]

Pultenaea

$P$. ferruginea var. deanei add: SC

$P$. vrolandii add: SWS

Sesbania

S. cannabina var. cannabina add: NWS

Tephrosia

$T$. grandiflora add: ${ }^{\dagger} \mathrm{SC}$
Trifolium

T. alexandrinum add: ${ }^{\dagger} \mathrm{NC},{ }^{\dagger} \mathrm{CC},{ }^{\dagger} \mathrm{NWS}$

$T$. fragiferum add: *SWP

FLACOURTIACEAE

add: DOVYALIS E. Mey.

*D. caffra Warb.; ${ }^{\dagger} \mathrm{CC} ; \mathrm{S}$ Afr

Xylosma

add: X. terrae-reginae White \& Sleumer; $\mathrm{NC} ; \mathrm{Q}$

\section{FRANKENIACEAE}

Frankenia

add: F. crispa J. M. Black; SFWP; VS

GERANIACEAE

Geranium

$G$. homeanum add: NT, ST, NWS

$G$. neglectum add: SC

\section{GOODENIACEAE}

Goodenia

$G$. elongata add: SWS

$G$. varia add: ?SWP

\section{GYROSTEMONACEAE}

Codonocarpus

correct to: $C$. codnifolius

Gyrostemon

G. australasicus correct to: (Moq.) Heimerl

HYPERICACEAE

Hypericum

H. japonicum add: SWS

\section{LAMIACEAE}

Mentha

M. pulegium add: ${ }^{\dagger} \mathrm{NC}$

Plectranthus

add: P. apreptus S. T. Blake; NC; Q

add: $P$. argentatus S. T. Blake; NC; Q

Prostanthera

add: P. cruciflora J. H. Willis; NWS, NT

Salvia

$S$. reflexa add: ${ }^{\dagger} \mathrm{SC}$

$S$. verbenaca add: ${ }^{\dagger} \mathrm{CC}$

\section{LAURACEAE}

Beilschmiedia

B. obtusifolia change authors to: (F. Muell. ex Meisn.) F. Muell.

Cassytha

C. glabella delete: $\mathrm{CT}$

C. melantha delete: NWP

delete: $C$. paniculata

delete: C. phaeolasia

add: C. racemosa Nees; NC; Q

LORANTHACEAE

Muellerina

$M$. eucalyptoides add: SWS

\section{MALVACEAE}

Anoda

A. cristata add: ${ }^{\dagger} \mathrm{NWP}$

Malva

$M$. nicaeënsis add: ${ }^{\dagger}$ SWS, ${ }^{\dagger}$ ?SWP

Malvastrum 
M. coromandelianum add: $\mathrm{CC}$

\section{MENISPERMACEAE}

delete: Fawcettia

add: PLEOGYNE Miers

P. australis Benth.; NC; QW

Tinospora

add: T. tinosporoides (F. Muell.) Forman;

$\mathrm{NC} ; \mathrm{Q}$

[Fawcettia tinosporoides F. Muell.]

\section{MIMOSACEAE}

To be treated as the subfamily Mimosoideae of the family FABACEAE

Acacia

$A$. decora add: SWP

A. falcata add: CWS; delete SWS

A. juncifolia var. planifolia add: NT

add: A. melvillei Pedley; SFWP

$A$. paradoxa add: SWP

A. pravissima add: SC

A. wilhelmiana add: NFWP

\section{MYOPORACEAE}

Myoporum

M. montanum add: CWS

\section{MYRTACEAE}

Eucalyptus

add: E. baxteri (Benth.) Maiden \& Blakely; SC;

VS; MOHCA

correct to: $E$. caliginosa

add: LOPHOSTEMON Schott

L. confertus (R. Br.) Peter G. Wilson \&

Waterhouse; $\mathrm{NC},{ }^{\dagger} \mathrm{CC} ; \mathrm{Q}$

[Tristania conferta $\mathrm{R}$. Br.]

L. suaveolens (Soland. ex Gaertn.) Peter G.

Wilson \& Waterhouse; CC, CT

[Melaleuca suaveolens Soland. ex Gaertn.,

Tristania suaveolens (Soland. ex Gaertn.) Sm.]

Melaleuca

delete: $M$. halmaturorum

Tristania

delete: $T$. conferta

delete: $T$. laurina

delete: $T$. suaveolens

add: TRISTANIOPSIS Brongn. \& Gris.

T. collina Peter G. Wilson \& Waterhouse; NC, CC, SC, CT, ST; $Q$

T. laurina (Sm.) Peter G. Wilson \&

Waterhouse; NC, CC, SC; QV

[Melaleuca laurina Sm., Tristania laurina

(Sm.) R. Br.]

\section{OLEACEAE}

Notelaea

N. microcarpa var. microcarpa add: ST

Olea

$O$. africana add: ${ }^{\dagger} \mathrm{CWS}$

\section{ONAGRACEAE}

Ludwigia

L. octovalvis add: SWP

OROBANCHACEAE

Orobanche

$O$. minor add: ${ }^{\dagger}$ SWP

\section{OXALIDACEAE}

Oxalis

$O$. articulata change distributions to: ${ }^{\dagger} \mathrm{NC}$,

${ }^{\dagger} \mathrm{CC},{ }^{\dagger} \mathrm{ST},{ }^{\dagger} \mathrm{SWS}$; V; S Amer

$O$. bowie $i$ add: ${ }^{\dagger} \mathrm{SWS}$; $\mathrm{V}$; delete: SWP

$O$. brasiliensis add: ${ }^{\mathrm{NC}}$, ${ }^{\dagger} \mathrm{SWS}$

add: O. chnoödes Lourt.; NC, CC, SC; Q; NG

$O$. corniculata change distributions to: ${ }^{\dagger} \mathrm{C}$,

SC; QVS; ?S. Amer

delete: $O$. corymbosa

add: $O$. debilis Kunth

var. corymbosa (DC.) Lourt.; ${ }^{\dagger} \mathrm{LHI}, \mathrm{CC}, \mathrm{SC}$;

VS; S Amer

[O. corymbosa DC.]

add: O. exilis A. Cunn.; NC, CC, SC, NT, CT, ST, CWS, SWS; QVT; NZ

$O$. incarnata delete: SC, SWS; add: CWS

add: *O. mallobolba Cav.; ${ }^{\dagger}$ CWS; S Amer

add: O. perennans Haw.; NC, CC, CT, ST, NWS, CWS, SWS, NWP, SWP NFWP, SFWP; VS; S Afr

$O$. pes-caprae add: ${ }^{\dagger} \mathrm{NFWP},{ }^{\dagger} \mathrm{SFWP}$

$O$. purpurea change distribution to: ${ }^{\dagger} \mathrm{CC},{ }^{\dagger} \mathrm{CT}$, TST; VSW; S Afr

add: O. radicosa A. Rich.; LHI, NC, CC, SC,

NT, CT, ST, NWS, CWS, SWS, NWP:

QVS; Asia, Afr

add: O. rubens Haw.; LHI, NC, CC, SC;

QVTS; NZ

add: O. sp.; CC, CT, NWS; V; ?NZ

\section{PASSIFLORACEAE}

Passiflora

P. mollissima add: ${ }^{\dagger} \mathrm{CC}$

\section{PITTOSPORACEAE}

Bursaria

$B$. longisepala add: ST

\section{PLANTAGINACEAE}

Plantago

$P$. coronopus ssp. coronopus add: ${ }^{\dagger} \mathrm{CT}$

POLYGONACEAE

add: *FALLOPIA Adans.

${ }^{*} \mathrm{~F}$. convoluulus (L.) A. Löve; ${ }^{\dagger} \mathrm{NC},{ }^{\dagger} \mathrm{CC},{ }^{\dagger} \mathrm{SC}$,

${ }^{\dagger} \mathrm{NT},{ }^{\dagger} \mathrm{CT},{ }^{\dagger} \mathrm{ST},{ }^{\dagger} \mathrm{NWS} ;{ }^{\dagger} \mathrm{CWS},{ }^{\dagger} \mathrm{SWS}$,

†NWP, †SWP; W; Eur, Asia, Afr, Amer

[Polygonum convolvulus $\mathbf{L}$.]

add: PERSICARIA Mill.

P. attenuata (R. Br.) Soják; NC, NWP, SFWP; QYSW

[Polygonum attenuatum $\mathrm{R}$. Br.]

*P. capitata (Hamilton ex D. Don) Gross; ${ }^{\dagger} \mathrm{NC}$, †CC; Asia

[Polygonum capitatum Hamilton ex D. Don]

P. dichotoma (Blume) Masam.; NC; Q; Asia, S Afr

[Polygonum dichotomum Blume]

P. elatior (R. Br.) Soják; NC, CC, SC; Q

[Polygonum elatius $\mathrm{R}$. Br.]

P. hydropiper (L.) Spach; NC, CC, SC, NT, CT, ST, NWS, CWS, SWS, SWP; QV;

Eur, Asia, Amer

[Polygonum hydropiper L.]

P. lapathifolia (L.) S. F. Gray; NC, CC, SC, NT, CT, ST, NWS, CWS, SWS, NWP, SWP, NFWP, SFWP; QVTYS; Eur, Asia, Amer

[Polygonum lapathifolium L.]

P. orientalis (L.) Spach; NC, CC, SC, NWS, 
CWS, SWS, NWP, SWP; QY; India, Asia [Polygonum orientale L.]

*P. maculata (Rafin.) Löve \& Löve; †SC, †CT, †ST; VT; Eur [Polygonum persicaria L.]

P. prostrata (R. Br.) Soják; CC, NT, CT, ST, NWS, CWS, SWS, NWP, SWP; QVTYSW; NZ [Polygonum prostratum $\mathrm{R}$. Br.]

P. strigosa (R. Br.) Gross; NC, CC, CT; Q; India, China [Polygonum strigosum $\mathrm{R}$. Br.]

P. sp. A; NC, CC, SC, NT, CT, ST, NWS, SWP; QVTYSW; NZ

[Polygonum decipiens $\mathrm{R}$. Br.]

P. sp. B; NC, CC, SC, NT, CT, ST, SWS; VT; India

[Polygonum praetermissum Hook. f.]

P. sp. C.; NC, CC, SC; QVTW; N Caled [Polygonum subsessile R. Br., Polygonum minus ssp. subsessile ( $\mathrm{R}$. $\mathrm{Br}$.) Danser]

Polygonum

delete: $P$. attenuatum

delete: $P$. capitatum

delete: $P$. convolvulus

delete: $P$. cuspidatum

delete: $P$. decipiens

delete: $P$. dichotomum

delete: $P$. elatius

delete: $P$. hydropiper

delete: $P$. lapathifolium

delete: $P$. orientale

add: *P. patulum Bieb.; ${ }^{\dagger} \mathrm{NWS},{ }^{\dagger} \mathrm{CWS},{ }^{\dagger}$ SFWP.

VS; Eur

delete: $P$. persicaria

$P$. plebeium add: CWS

delete: $P$, praetermissum

delete: $P$. prostratum

delete: $P$. sachalinense

delete: $P$. strigosum

delete: $P$. subsessile

add: *REYNOUTRIA Houtt.

*R. japonica Houtt.; ${ }^{\text {SWWS}}$; Japan

[Polygonum cuspidatum Siebold \& Zucc.]

* R. sachalinensis (F. Schmidt) Nakai; ${ }^{\dagger} \mathrm{CT}$;

V; Asia, Eur

[Polygonum sachalinense F. Schmidt]

PORTULACACEAE

Calandrinia

add: C. granulifera Benth.; SWS, SWP; VW

[C. neesiana $\mathrm{Hj}$. Eichler, C. pygmaea $\mathrm{F}$.

Muell.]

delete: C. neesiana

\section{PRIMULACEAE}

Lvsimachia

L. vulgaris var. davurica

correct author to: (Ledebour) R. Knuth

\section{PROTEACEAE}

Banksia

add: B. aemula R. Br.; NC, CC, Q

[B. serratifolia Salisb.]

delete: $B$. aspleniifolia

add: B. conferta $A$. S. George var. penicillata

A. S. George; CT

$B$. marginata add: SWP

add: B. oblongifolia Cav.; NC, CC; Q

[B. aspleniifolia Salisb., B. robur var. minor Maiden \& Camfield] delete: $B$. serratifolia

Grevillea

add: G. sp. aff. aspleniifolia; NT

add: G. sp. aff. miqueliana; NT; Q

\section{RANUNCULACEAE \\ Clematis}

C. fawcettii delete: NT

\section{RHAMNACEAE}

Pomaderris

$P$. discolor add: $\mathrm{SC}$

$P$. eriocephala add: SC

$P$. prunifolia add: CWS

\section{RUBIACEAE}

Asperula

A. scoparia delete: var. scoparia, var. ulicina, add to synonymy: $A$. scoparia var. ulicina Airy Shaw \& Turrill

Richardia

add: *R. scabra L.; ${ }^{\dagger} \mathrm{NC} ;$ Amer, Afr

\section{RUTACEAE}

Acronychia

add: A. sp.; NC

Boronia

$B$. barkeriana delete: $\mathrm{NC}$

$B$. ledifolia add: ST

$B$. parviflora add: NC

$B$. repandra correct to: repanda

$B$. thujona add: NC

Eriostemon

E. trachyphyllus add: CWS

SAPOTACEAE

Amorphospermum

add: A. whitei Aubr.; NC; Q

Niemeyera

delete: $N$. prunifera

\section{SCROPHULARIACEAE}

Bacopa

add: *B. amplexicaulis (Pursh) Wattst.; ${ }^{*} \mathrm{NC}$; Amer

Buchnera

$B$. gracilis add: NWS

delete: $B$. urticifolia

Kickxiā

$K$. sieberi add: ${ }^{\dagger} \mathrm{NWP},{ }^{\dagger} \mathrm{NFWP}$

Linaria

$L$. arvensis add: SWP

delete: $L$. dalmatica

L. genistifolia change to:

L. genistifolia ssp. genistifolia

add: ssp. dalmatica (L.) Maire \& Petitmergin; ${ }^{\dagger} \mathrm{NT}$ †CWS, ${ }^{\dagger} \mathrm{SWP}$; Eur

[L. dalmatica (L.) Miller]

$L$. pelisseriana add: ${ }^{\dagger} \mathrm{NWS},{ }^{\dagger} \mathrm{SWP}$

Veronica

V. persica add: CWS

SOLANACEAE

deleté: Anthocercis

Capsicum

add: ${ }^{*}$ C. annuum L. var. glabriusculum (Dunal) Heiser \& Prickersgill; ${ }^{\text {NWWS }}$ QY; Mexico 
add: CYPHANTHERA Miers

C. anthocercidea (F. Muell.) Haegi; ?CC; V [Anthocercis frondosa (Miers) J. M. Black, $A$. eadesii F. Muell.]

C. albicans (A. Cunn.) Miers

[Anthocercis albicans A. Cunn.]

ssp. albicans; CC, NT, CT, ST, CWS; QV [C. ovalifolia Miers]

ssp. tomentosa (Benth.) Haegi; CWS, SWS, NWP, SWP

[Anthocercis albicans var. tomentosa Benth.] ssp. notabilis Haegi; NWS

[Anthocercis albicans sensu Maiden \& Betche]

C. scabrella (Benth.) Miers; CC, CT [Anthocercis scabrella Benth.]

Physalis

delete: $P$. angulata

add: *P. ixocarpa Brot. ex Hornem.; $\uparrow$ LHI

${ }^{\dagger} \mathrm{NC},{ }^{\dagger} \mathrm{CC},{ }^{\dagger} \mathrm{NT},{ }^{\dagger} \mathrm{NWS},{ }^{\dagger} \mathrm{CWS},{ }^{\dagger} \mathrm{NWP}$;

$\mathrm{Q}$; Amer

add: *P lanceifolia Nees; ${ }^{\dagger}$ NWP; Q; Amer

delete: $P$. longifolia - leave as $P$. minima

$P$. virginiana delete: $\mathrm{f}$ macrophysa add: var. sonorae (Torrey) Waterfall; ${ }^{\dagger}$ NWS,

$\uparrow \mathrm{CWS}$ †SWP; Q; N Amer

add: *P viscosa $\mathrm{L} . ;{ }^{\dagger} \mathrm{SWS},{ }^{\dagger} \mathrm{SWP} ; \mathrm{QVSW}$; Amer

Solanum

$S$. linearifolium add: NT

$S$. rostratum add: ${ }^{\dagger} \mathrm{NC}$

\section{STERCULIACEAE}

Rulingia

add: R. dasyphylla (Andr.) Sweet; NC, CC, SC, NT, CT, NWS, CWS; QV

[R. pannosa $R$. Br.]

delete: $R$. pannosa

\section{THYMELAEACEAE}

Pimelea

$P$. axiflora

replace ssp. A by: ssp. axiflora

replace ssp. B by: ssp. alpina (F. Muell. ex Benth.) Threlfall

add: P. bracteata Threlfall; ST

$[P$. ligustrina var. glabra Maiden \& Betche,

$P$. sp. B sensu Jacobs \& Pickard]

$P$. curviflora

replace $s s p$. A by: ssp. curviflora

replace ssp. B by: ssp. micrantha $(F$. Muell. ex

Meisn.) Threlfall

replace ssp. C by: ssp. gracilis ( $R . B r$.) Threlfall [P. gracilis $\mathrm{R} . \mathrm{Br}$.]

replace var. A by: var, gracilis

replace var. $B$ by: var. sericea Benth.

replace var. $C$ by: var. divergens Threlfall

replace var. $D$ by: var. subglabra Threlfall

replace var. E by: var. acuta Threlfall

add: P. elongata Threlfall; NWP; QS

$P$. flava

replace $\mathrm{ssp}$. B by: $\mathrm{ssp}$. dichotoma (Schlecht.)

Threlfall

\section{P. latifolia}

replace ssp. A by: ssp. latifolia

replace ssp. B by: ssp. altior (F. Muell.)

Threlfall

$[P$. altior F. Mueli., $P$. altior var. typica

Domin]

replace var. A by: var. altior

replace var. B by: var. parvifolia Domin

replace ssp. C by: ssp. hirsuta (Meisn.)

Threlfall

replace ssp. D by: ssp. elliptifolia Threlfall

$P$. ligustrina

replace $s s p$. A by: ssp. ligustrina

$[P$. elata F. Muell. ex Meisn.]

replace $s s p$. B by: ssp. ciliata Threlfall

replace ssp. $C$ by: ssp. hypericina (A. Cunn. ex Hook.) Threlfall

$P$. linifolia

replace ssp. A by: ssp. linifolia

replace ssp. B by: ssp. collina (R. Br.) Threlfall replace $s s p$. C by: ssp. linoides (A. Cunn.)

Threlfall

replace ssp. D by: ssp. caesia Threlfall

$P$. microcephala

replace $\mathrm{ssp}$. A by: $\mathrm{ssp}$. microcephala

add: P. neo-anglica Threlfall: NT, NWS; Q

$[P$. sp. C sensu Jacobs \& Pickard]

P. octophylla

replace ssp. B by: ssp. ciliolaris Threlfall

P. simplex

replace ssp. A by: ssp. simplex

replace ssp. B by: ssp. continua (J. M. Black)

Threlfall

add: $P$. venosa Threlfall; NT, NWS

delete: species $A$ to $D$

URTICACEAE

add: AUSTRALINA Gaudich.

A. pusilla Gaudich.; NC, SC, NT, CT, ST; VT [A. muelleri Wedd.]

\section{VERBENACEAE}

Phyla

$P$. nodiflor a add: ${ }^{\dagger} \mathrm{CWS}$

\section{VISCACEAE}

Korthalsella

delete: $K$. japonica

add: K. rubra (Tiegh.) Endl.

ssp. rubra; LHI, NC, CC, SC, NT, CT, ST; QV; $\mathrm{NG}$

[K. howensis (Tiegh.) Endl., K. opuntia auct. non (Thunb.) Merr., $K$. japonica auct. non

(Thunb.) Engl., $K$. brassiana auct. non

Blakely]

ssp. geijericola Barlow; NWS, CWS, SWS, NWP, SWP; Q

\section{WINTERACEAE}

Tasmannia

$T$. insipida correct synonym to: $T$. dipetala

$T$. lanceolata add: SC; delete: NT

T. xerophila delete: NC, NT

Manuscript received 16.3.1983 